\title{
Unusual complications of thyroid carcinoma
}

\author{
P J D Owen, J H Lazarus, R E Morse
}

Postgrad Med J 2003;79:55-56

Thyroid carcinomas are the most frequent endocrine malignancies. Complications may arise from an established malignancy and these may lead to the initial clinical presentation or cause subsequent problems. In this case report two elderly patients with differentiated and undifferentiated thyroid carcinomas who suffered unusual medical complications are described. The incidence and treatment of thyroid carcinoma is discussed and the importance of fine needle aspiration of thyroid nodules and the recognition of iodine containing radiographic contrast media in the causation of iodine induced thyrotoxicosis is highlighted.

$\mathrm{T}$ hyroid carcinomas are the most frequent endocrine malignancies, the incidence increasing with advancing age, ${ }^{1}$ with approximately 1000 new cases per annum in England and Wales. ${ }^{2}$ Complications can arise from an established malignancy and these may lead to the initial clinical presentation or cause subsequent problems.

Primary differentiated thyroid carcinoma (follicular, papillary, or mixed) accounts for over $90 \%$ of all thyroid malignant tumours ${ }^{3}$ and has an overall excellent prognosis. However this prognosis worsens when the disease is diagnosed in elderly patients as poor prognostic features are often evident at the time of diagnosis, ${ }^{4}$ and the risk of tumour recurrence also increases after the age of 60 years.

Undifferentiated and medullary thyroid carcinomas are rare; anaplastic carcinoma is usually rapidly progressive and fatal and again more prevalent in the elderly population.

We describe two cases of thyroid carcinoma occurring in elderly patients who suffered unusual medical complications.

\section{CASE REPORTS}

\section{Case 1}

A 91 year old woman presented as an emergency with an episode of syncope resulting in a fall. On examination she was sweaty with a bradycardia of 30 beats/min and blood pressure measuring 60/30 mm Hg. A firm, non-tender irregular mass was palpable in the left side of her neck measuring $6 \times 4 \times 3$ $\mathrm{cm}$. She was initially treated with atropine $(1 \mathrm{mg})$ with resolution of her bradycardia to 60 beats/min and restoration of blood pressure (110/60 mm Hg).

Three weeks before admission she had presented to the ear, nose, and throat department with a six week history of a progressive painful neck mass associated with hoarseness.

Investigations at that time included normal thyroid function tests but an ultrasound scan of the neck showed a large hypoechoic mass arising from the left lobe of the thyroid associated with enlarged lymph nodes in the left side of the neck. Computed tomography of the neck and thorax revealed extension of the mass into the mediastinum encasing the major vascular structures and causing deviation and compression of the trachea. Fine needle aspiration of the thyroid mass revealed cells with appearances typical of anaplastic thyroid carcinoma.
During her inpatient stay she experienced recurrent bradycardic and hypotensive episodes associated with both syncopal and presyncopal symptoms. A permanent dual chamber pacemaker was inserted with no significant symptomatic improvement. Further therapeutic measures included fludrocortisone and low dose $\beta$-blocker therapy both of which provided no symptomatic improvement.

Unfortunately her condition continued to deteriorate with persistent hypotensive episodes and six weeks subsequent to her initial presentation she died.

\section{Case 2}

An 81 year old man was referred with a history of an enlarging neck mass associated with weight loss, palpitations, and sweating. Thyroid function tests confirmed the clinical diagnosis of thyrotoxicosis. Ultrasound scanning of the mass revealed a heterogeneous appearance and a large hyperplastic nodule.

${ }^{123}$ I scanning on three separate occasions demonstrated an abnormally low uptake ( $8 \%$ at four hours) and he was not given radioiodine treatment. Further history revealed the patient had received an iodine contrast enema four weeks before his referral for investigation into weight loss that showed no abnormality. His presenting features resolved and his thyroid function tests normalised without treatment.

During follow up 12 months after his initial referral he was noted to have a lesion on his anterior chest wall, and an ultrasound scan of this lesion demonstrated its erosion through the sternum. Fine needle aspiration showed cells consistent with metastatic follicular carcinoma.

After referral to the oncologists he received palliative external radiotherapy to the sternal mass. Subsequent serum thyroglobulin measurements remained markedly raised and he was maintained on a suppressive dose of thyroxine.

Unfortunately he developed further metastases and he died eight months after his diagnosis.

\section{DISCUSSION}

Both these cases represent unusual complications of thyroid carcinoma.

Although thyroid carcinoma is rare, its occurrence representing $0.5 \%-1.0 \%$ of all human malignant tumours, it is still the most frequent endocrine malignant neoplasm. ${ }^{3}$ Signs at presentation may reflect primary tumour growth such as an enlarged neck mass or metastatic spread with lymph node involvement. Other features may include those of concurrent hyperthyroidism. ${ }^{6}$ Local invasion of surrounding structures may cause dysphagia or hoarseness.

Follicular carcinoma accounts for around a fifth of all thyroid cancers with a sex distribution showing a strong predominance of women compared with men, ${ }^{8}$ with long term survival rates exceeding $90 \%{ }^{9}$; however, recurrence is common.

Anaplastic thyroid carcinoma is a rare, highly malignant tumour of elderly people that is resistant to treatment, ${ }^{10}$ and as one of the most aggressive solid tumours known is usually associated with a rapidly fatal clinical evolution. ${ }^{11}$ 


\section{Learning/summary points}

- Incidence of thyroid carcinoma increases with age.

- Thyroid carcinoma is the commonest endocrine malignancy.

- Differentiated thyroid carcinoma has an overall good prognosis.

- Fine needle aspiration biopsy of thyroid nodules is rapid and cost effective.

- lodine contamination is an important consideration in elderly patients.

- Ultrasonography and computed tomography are useful in the management of thyroid cancer.

Although the incidence of distant metastases is very high, outcome is usually dependent upon regional recurrence and spread. Although symptomatic bradycardia has been previously reported, ${ }^{12}$ to our knowledge this is the first case illustrating how infiltrative carcinoma of thyroid origin involving the glossopharyngeal and vagus nerves resulting in neuralgia and vasodepressor syncope and subsequent pacemaker insertion.

Presyncopal and syncopal symptoms are distressing and treatment is worth considering. The treatment (of this specific presentation) may be both specific and generalised. Specific treatment options include pacemaker implantation for bradycardia (but in this case vagal stimulation was so intense that it could not be overcome), or vagotomy may be another option. Other adjunctive therapies as used in our case involve low dose $\beta$-blockade, which may limit reflex vagal stimulation by reducing intramyocardial pressure. ${ }^{13}$ Fludrocortisone may also be considered.

Bone metastases have been documented at the time of initial diagnosis in up to $47 \%$ of patients with thyroid carcinoma, ${ }^{14}$ vertebral deposits being commonest and adversely affecting prognosis.

The importance of radiographic contrast agents causing iodine induced thyrotoxicosis is highlighted in the second case and it is advisable that caution is observed in the administration of iodine-containing drugs especially iodinated contrast medium in patients with pre-existing thyroid disease. ${ }^{15}$

Generalised treatment of thyroid carcinoma may be directed towards reducing tumour mass with aggressive multimodality combination regimens of surgery with or without radiotherapy/chemotherapy providing some benefit. An aggressive approach to management of differentiated thyroid carcinoma is likely to render about $90 \%$ of patients permanently free of disease. ${ }^{5}$ However in anaplastic carcinoma, due to the aggressive and progressive nature of the malignancy, therapeutic success is often limited (resulting in poor survival rates) and mean survival after diagnosis is six to 12 months.

\section{CONCLUSION}

In summary we present two cases of thyroid carcinoma in elderly patients illustrating the unusual complications of bradycardia and sternal metastasis. We also note that the use of fine needle aspiration biopsy in the evaluation of thyroid nodules provides a rapid and cost effective method of investigation. The importance of an excess iodine load (in our case secondary to radiographic contrast material) must be considered in patients presenting with thyrotoxicosis as increasing numbers of radiological investigations with contrast are performed on an ageing population who may have underlying thyroid disease.

\section{Authors' affiliations \\ P J D Owen, J H Lazarus, R E Morse, Department of Medicine, University Hospital of Wales, Cardiff}

Correspondence to: Dr P J D Owen, Department of Medicine, University of Wales College of Medicine, Heath Park, Cardiff CF14 4XN, UK; Lazarus@cf.ac.uk

Submitted 17 May 2002

Accepted 14 October 2002

\section{REFERENCES}

1 Schlumberger M, Pacini F. Features of thyroid cancer. Thyroid tumors. Paris: Editions Nucleon, 1997: 49 (chapter 3).

2 Jones MK. Management of nodular thyroid disease. BM 2001;323:293-4.

3 Maussier ML, Danese D, D'Errico G, et al. Clinical and laboratory follow up in differentiated thyroid carcinoma. Rays 2000;25:239-44. follow up in differentiated thyroid carcinoma. Rays 2000;25:239-
van Tol KM, de Vries EG, Dullart RP, et al. Differentiated thyroid carcinoma in the elderly. Crit Rev Oncol Hematol 2001;38:79-9 5 Mazzaferri EL. Long term outcome of patients with differentiated thyroid carcinoma: effect of therapy. Endocrine Practice 2000;6:469-76.

6 Ruggieri M, Scocchera F, Genderini M, et al. Hyperthyroidism and concurrent thyroid carcinoma. European Review for Medical and Pharmacological Sciences 1999:3:265-8.

7 Inabet WB. Surgical management of thyroid cancer. Endocrine Practice 1999:6:465-8.

8 Tasca C. Histopathology of thyroid carcinoma. A review of 500 cases. Morphologie et Embryologie 1978;24:331-6.

9 Mazzaferri EL. An overview of the management of papillary and follicular thyroid carcinoma. Thyroid 1999;9:421-7.

10 Voutilainen PE, Multanan M, Haapiainen RK, et al. Anaplastic thyroid carcinoma survival. World J Surg 1999;23:975-8.

11 Mai DD, Mai KT, Shamji FM. Fine needle aspiration biopsy of anaplastic thyroid carcinoma: a case report. Acta Cytol 2001;45:761-4.

12 Demeter JG, De Jong SA, Lawrence AM, et al. Anaplastic thyroid carcinoma: risk factors and outcome. Surgery 1991;110:956-61.

13 Calkins H, Douglas PZ. Hypotension and syncope. Heart disease. A tex book of cardiovascular medicine. Vol 1. (Braunwald.) Philadelphia: W B Saunders, 1997: 932-9 (chapter 27)

14 Pittas AG, Adler M, Fazzari M, et al .Bone metastases from thyroid carcinoma: clinical characteristics and prognostic variables in one hundred and forty six patients. Thyroid 2000;10:261-8.

15 Chen CC, Huang WS, Huang SC, et al. Thyrotoxicosis aggravated by iodinated contrast medium: a case report. Chung Hua I Hsueh Tsa Chih 1994;53:379-82.
1994: 